\title{
BOUNDARY BEHAVIOR OF POSITIVE SOLUTIONS OF THE HEAT EQUATION ON A SEMI-INFINITE SLAB
}

\author{
B. A. MAIR
}

\begin{abstract}
In this paper, the abstract Fatou-Naim-Doob theorem is used to investigate the boundary behavior of positive solutions of the heat equation on the semi-infinite slab $X=\mathbf{R}^{n-1} \times \mathbf{R}_{+} \times(0, T)$. The concept of semifine limit is introduced, and relationships are obtained between fine, semifine, parabolic, one-sided parabolic and two-sided parabolic limits at points on the parabolic boundary of $X$. A Carleson-Calderón-type local Fatou theorem is also obtained for solutions on a union of two-sided parabolic regions.
\end{abstract}

0. Introduction. The boundary behavior of positive solutions of second-order parabolic equations on a horizontal boundary has been studied in [10] by applying the abstract Fatou-Naim-Doob theorem (cf. [13]). The main aim of this paper is to apply the same methods to investigate the boundary behavior of positive solutions on a vertical boundary. This subject has already been studied by classical methods in [5-8, and 14].

This paper obtains the Calderón-type result (Theorem A) in [14] and special cases of results in $[\mathbf{7}, \mathbf{8}]$ by means of fine convergence. The advantage of using this method is that, although the results in this paper are for solutions of the heat equation, it is clear (cf. [10]) that if a suitable integral representation is obtained then the results in $\S \S 2,3,6$, and 7 still hold for positive solutions of more general parabolic equations on the semi-infinite slab $X=\mathbf{R}^{n-1} \times \mathbf{R}_{+} \times(0, T)$. In particular the Calderón-type local Fatou theorem with two-sided parabolic approach regions (Theorem 7.3) would still hold (cf. [5]).

Although the main interest is in behavior at the vertical boundary, this paper does not consider only the right half-space, but rather the semi-infinite slab $X$, this obtaining results for both horizontal and vertical boundaries. To do this, new nonsemithin sets are obtained in $\S 3$ and new Harnack inequalities are found in $\S 4$.

I would like to thank Professor J. C. Taylor of McGill University, Montréal, for his continuing interest in my work.

1. Preliminaries. Throughout this paper, $0<T \leq \infty$ and $n \in \mathbf{N}$ are fixed. $X$ denotes the semi-infinite slab

$$
\begin{aligned}
& \mathbf{R}^{n-1} \times \mathbf{R}_{+} \times(0, T)=\left\{\left(x^{\prime}, x_{n}, t\right): x^{\prime} \in \mathbf{R}^{n-1}, x_{n}>0,0<t<T\right\}, \\
& H=\mathbf{R}^{n-1} \times \mathbf{R}_{+} \times\{0\}, \quad V=\mathbf{R}^{n-1} \times\{0\} \times[0, T) \text { and } B=H \cup V .
\end{aligned}
$$

Then $B$ is the parabolic boundary of $X$.

Received by the editors May 7, 1985. Paper presented at the 91st Annual Meeting of the AMS, January 12, 1985.

1980 Mathematics Subject Classification. Primary 31B25; Secondary 35K20, 35K05.

Key words and phrases. Fine limit, semifine limit, parabolic limit, two-sided parabolic limit. 
The fundamental solution for the heat equation $\Delta_{x} u=\partial u / \partial t$ on $\mathbf{R}^{n} \times \mathbf{R}$ is given by,

$$
W(x, t ; y, s)= \begin{cases}{[4 \pi(t-s)]^{-n / 2} \exp \left(-\frac{|x-y|^{2}}{4(t-s)}\right),} & \text { if } t>s \\ 0, & \text { if } t \leq s\end{cases}
$$

Define

$$
G(x, t ; y, s)=W(x, t ; y, s)-W\left(x, t ;\left(y^{\prime},-y_{n}\right), s\right) .
$$

For each $(x, t)=\left(x^{\prime}, x_{n}, t\right) \in X, b \in B$, define,

$$
K_{b}(x, t)= \begin{cases}G\left(x, t ;\left(b^{\prime}, b_{n}\right), 0\right), & \text { if } b=\left(b^{\prime}, b_{n}, 0\right) \in H, \\ \left.\frac{\partial}{\partial b_{n}} G\left(x, t ;\left(b^{\prime}, b_{n}\right), s\right)\right|_{b_{n=0}}, & \text { if } b=\left(b^{\prime}, 0, s\right) \in V .\end{cases}
$$

Now, it is well known that the solutions of the heat equation generate a strong harmonic space on $X$ (cf. [1]). In [11], axiomatic potential theory and Martin's method for the construction of ideal boundaries are used to obtain the following integral representation theorem.

THEOREM 1.1. Let $u \geq 0$ be a solution of the heat equation on $X$. Then there exists a unique Borel measure $\mu$ on $B$ such that

$$
u(x, t)=\int K_{b}(x, t) d \mu(b), \quad \text { for all }(x, t) \in X .
$$

This $\mu$ is called the representing measure for $u$.

The following abstract Fatou-Naim-Doob theorem then follows from [13].

THEOREM 1.2. Let $u>0, v>0$ be solutions of the heat equation on $X$, represented by measures $\mu, \nu$ respectively. Then $u / v$ has fine limit $d \mu / d \nu, \nu$-a.e. on $B$.

For any $E \subset X$ and $u \geq 0$, superharmonic on $X, R_{E} u$ denotes the reduced function of $u$ on $E$.

For each $b \in B, \mathcal{F}(b)$ denotes the fine filter at $b$. For each $b \in B$, let $X_{b}^{+}=$ $\left\{(x, t) \in X: K_{b}(x, t)>0\right\}$. Then $X \backslash X_{b}^{+}$is empty if $b \in H$ and nonempty if $b \in V$. However, $K_{b}=0$ on $X \backslash X_{b}^{+}$if $b \in V$; hence $X \backslash X_{b}^{+}$is thin at each $b \in B$.

Throughout this paper, $C$ denotes a general positive constant (not necessarily the same at different occurrences) which may depend on $n$ and other constants.

PROPOSITION 1.3. If $E$ is thin at $b$, then for any sequence $\left\{U_{m}\right\}$ of neighborhoods of $b$ in $\mathbf{R}^{n+1}$ decreasing to $\{b\}$ and $(x, t) \in X, \lim _{m \rightarrow \infty} \hat{R}_{E(m)} K_{b}(x, t)=0$, where $E(m)=E \cap U_{m}$.

Conversely, if there exist $(x, t) \in X_{b}^{+}$and a sequence $\left\{U_{m}\right\}$ as above such that $\lim _{m \rightarrow \infty} \hat{R}_{E(m) \cap X_{b}^{+}} K_{b}(x, t)=0$, then $E$ is thin at $b$.

PROOF. This result follows easily as in [10, Proposition 1.5] if the complement of any neighborhood of $b$ in $\mathbf{R}^{n+1}$ is thin at $b$. To see this, note the following.

(i) If $b=\left(b^{\prime}, b_{n}, 0\right) \in H$ and $\delta>0$, then $\left|x-\left(b^{\prime}, b_{n}\right)\right|>\delta$ or $t>\delta$ implies $K_{b}(x, t)<C t$, which is superharmonic on $X$ and tends to 0 as $(x, t) \rightarrow b$.

(ii) If $b=\left(b^{\prime}, 0, s\right) \in V$ and $\delta>0$, then $\left|x-\left(b^{\prime}, 0\right)\right|>\delta$ or $t-s>\delta$ implies $K_{b}(x, t) \leq C x_{n}$, which is superharmonic on $X$ and tends to 0 as $(x, t) \rightarrow b$. 
Hence, if $U$ is a neighborhood of $b \in B$ in $\mathbf{R}^{n+1}$, then $R_{X \backslash U} K_{b}(x, t) \rightarrow 0$ as $(x, t) \rightarrow b$, so $R_{X \backslash U} K_{b} \neq K_{b}$.

As stated before, this paper links fine convergence with certain types of geometric convergence at points of $B$. These regions will now be defined.

For each $b=\left(b^{\prime}, b_{n}, 0\right) \in H$ and $\alpha>0$,

$$
P(b ; \alpha)=\left\{(x, t) \in \mathbf{R}^{n} \times \mathbf{R}_{+}:\left|x-\left(b^{\prime}, b_{n}\right)\right|^{2}<\alpha t\right\}
$$

is called the parabolic region with aperture $\alpha$ and vertex $b$. For any $\delta>0$,

$$
P(b ; \alpha, \delta)=P(b ; \alpha) \cap\{(x, t): t<\delta\}
$$

is called a truncated parabolic region.

For each $b=\left(b^{\prime}, 0, s\right), \alpha>0, \beta>0, \delta>0$, $P(b ; \alpha: \beta)=\left\{\left(x^{\prime}, x_{n}, t\right): 0<\alpha(t-s)<\left|x-\left(b^{\prime}, 0\right)\right|^{2}<\alpha^{-1}(t-s), x_{n}>\beta\left|x^{\prime}-b^{\prime}\right|\right\}$ (here $\alpha<1$ ) is called the parabolic region with vertex $b$ and aperture $\alpha: \beta$.

$$
P(b ; \alpha: \beta, \delta)=P(b ; \alpha: \beta) \cap\left\{(x, t):\left|x-\left(b^{\prime}, 0\right)\right|<\delta\right\} .
$$

$T P(b ; \alpha: \beta)=\left\{\left(x^{\prime}, x_{n}, t\right):|t-s|<\alpha\left|x-\left(b^{\prime}, 0\right)\right|^{2}, x_{n}>\beta\left|x^{\prime}-b^{\prime}\right|\right\}$ is the two-sidedparabolic region with vertex $b$ and aperture $\alpha: \beta$.

$$
T P(b ; \alpha: \beta, \delta)=T P(b ; \alpha: \beta) \cap\left\{(x, t):\left|x-\left(b^{\prime}, 0\right)\right|<\delta\right\} .
$$

A real-valued function $f$ on $X$ is said to have parabolic limit $\lambda$ at $b \in B$ if $f$ converges to $\lambda$ within parabolic regions with vertex $b$.

As in [10], the parabolic filter at $b, \mathcal{P}(b)=\{E \subset X$ : for each $P(b ; \tau)$ there exists $\delta>0$ such that $P(b ; \tau, \delta) \subset E\}$ describes parabolic convergence at $b$.

2. Semifine and parabolic limits for arbitrary functions. As in [10], the semifine limit is introduced and its existence is shown to be a consequence of the existence of the parabolic limit for any function on $X$.

From now on fix $0<\gamma<1$.

Definition 2.1. (i) For each $b \in B, m \in \mathbf{N}$ define

$$
R_{m}(b)=\left\{\begin{array}{c}
\left.(x, t):\left|x-\left(b^{\prime}, b_{n}\right)\right|<\gamma^{m}, 0<t<\gamma^{2 m}\right\}, \quad \text { if } b=\left(b^{\prime}, b_{n}, 0\right) \in H, \\
\left\{(x, t):\left|x-\left(b^{\prime}, 0\right)\right|<\gamma^{m}, 0<t-s<\gamma^{2 m}, x_{n}>0\right\}, \\
\text { if } b=\left(b^{\prime}, 0, s\right) \in V . \\
J_{m}(b)=R_{m}(b) \backslash R_{m+1}(b) .
\end{array}\right.
$$

$J_{m}(b)$ will be denoted by $J_{m}$ when the context determines $b$.

(ii) A set $E \subset X$ is said to be semithin at $b \in B$ if there exists $(x, t) \in X_{b}^{+}$such that $\lim _{m \rightarrow \infty} \hat{R}_{E \cap J_{m}} K_{b}(x, t)=0$.

(iii) For each $b \in B, S(b)=\{E \subset X: X \backslash E$ is semithin at $b\}$ is the semifine filter at $b$. For any function $f$, semifine $\lim f(b)$ denotes the limit of $f$ along $S(b)$.

Theorem 2.2 (cf. [10, Proposition 2.4]). For each $b \in B, P(b) \subset S(b)$.

Proof. If $b=\left(b^{\prime}, b_{n}, 0\right) \in H$ define $u_{m}(x, t)=\int_{B_{m}} W(x, t ; y, 0) d y$, where

$$
B_{m}=\left\{y \in \mathbf{R}^{n}: \gamma^{m+2} \leq\left|y-\left(b^{\prime}, b_{n}\right)\right|<\gamma^{m-1}\right\} .
$$

Then as in [10, Proposition 2.4], $\inf _{J_{m}} u_{m}=\inf _{J_{1}} u_{1}>0$ for all $m$. Since $K_{b}(x, t) \leq$ $W\left(x, t ;\left(b^{\prime}, b_{n}\right), 0\right)$, the same estimates can be repeated here. 
Now, if $b=\left(b^{\prime}, 0, s\right) \in V$, define

$$
\begin{aligned}
& F_{m}=\left\{y=\left(y^{\prime}, 0, p\right):\left|y^{\prime}-b^{\prime}\right|<\gamma^{m-1},|p-s|<\gamma^{2(m-1)}\right\} \\
& B_{m}=F_{m} \backslash F_{m+3}, \quad u_{m}(x, t)=\int_{B_{m}} K_{y}(x, t) d y
\end{aligned}
$$

and consider the transformation

$$
\left(x^{\prime}, x_{n}, t\right) \rightarrow\left(\gamma^{-(m-1)}\left(x^{\prime}-b^{\prime}\right)+b^{\prime}, \gamma^{-(m-1)} x_{n}, \gamma^{-2(m-1)}(t-s)+s\right) .
$$

Then $\inf _{J_{m}} u_{m}=\inf _{J_{1}} u_{1}>0$. Now

$$
K_{b}(x, t) \leq C x_{n}(t-s)^{(r-n-2) / 2}\left|x-\left(b^{\prime}, 0\right)\right|^{-r}
$$

for all $(x, t)$, and $(x, t) \in J_{m}$ implies either $\gamma^{m+1} \leq\left|x-\left(b^{\prime}, 0\right)\right|<\gamma^{m}$ and $0<$ $t-s<\gamma^{2 m}$ or $\left|x-\left(b^{\prime}, 0\right)\right|<\gamma^{m}$ and $\gamma^{2(m+1)} \leq t-s<\gamma^{2 m}$. Hence

$$
\begin{aligned}
K_{b}(x, t) \leq C \gamma^{-(n+1)(m+1)} \min \left\{(t-s)^{-1 / 2}\left|x-\left(b^{\prime}, 0\right)\right|,\right. \\
\\
\left.(t-s)^{1 / 2}\left|x-\left(b^{\prime}, 0\right)\right|^{-1}, x_{n}\left|x^{\prime}-b^{\prime}\right|^{-1}\right\} .
\end{aligned}
$$

Let $0<\varepsilon<1$ and $X \backslash E \in P(b)$. Then there exists $m_{0}$ such that for $m \geq m_{0}$, $(x, t) \notin P\left(b ; \varepsilon^{2}: \varepsilon^{2}\right)$ for all $(x, t) \in E \cap J_{m}$. Then for $m \geq m_{0}$ and $(x, t) \in E \cap J_{m}$, $K_{b}(x, t) \leq C \gamma^{-(n+1)(m+1)} \varepsilon u_{m}(x, t)$. Fix $(x, t) \in X_{b}^{+}$such that $t-s>1$, then

$$
u_{m}(x, t) \leq C x_{n}\left(\text { volume of } B_{m}\right)=C \gamma^{(n+1)(m-1)} x_{n} \text {. }
$$

Hence $\hat{R}_{E \cap J_{m}} K_{b}(x, t) \leq C \varepsilon$, and so $E$ is semithin at $b$.

\section{Nonsemithin sets.}

Proposition 3.1 (CF. [10, Proposition 3.1]). Let $\left\{\left(y_{m}, t_{m}\right)\right\}$ converge to $b \in H$ within $P(b ; \alpha)$. Then for any $\beta>0, \bigcup_{m=1}^{\infty}\left\{\left(x, t_{m}\right) \in X:\left|x-y_{m}\right|^{2}<\beta t_{m}\right\}$ is not semithin at $b$.

PROOF. Let $y_{i, m}$ denote the $i$ th coordinate of $y_{m}$ and fix $(z, r) \in X$. Then there exists $m_{0}$ such that for all $m \geq m_{0}, t_{m}<r$ and $\sqrt{\beta t_{m}}<b_{n} / 4<b_{n} / 2<y_{n, m}$. Put

$$
E_{m}=\left\{\left(x, t_{m}\right) \in \mathbf{R}^{n} \times \mathbf{R}_{+}:\left|x-y_{m}\right|^{2}<\beta t_{m}\right\} .
$$

Then $E_{m} \subset X$ for all $m \geq m_{0}$, and it suffices to prove that $\bigcup_{m \geq m_{0}} E_{m}$ is not semithin at $b$. It is easy to see that $K_{b}\left(x, t_{m}\right) \geq C t_{m}^{-n / 2}$ for all $\left(x, t_{m}\right) \in E_{m}$, and $\hat{R}_{E_{m}} 1$ dominates the solution to the Dirichlet problem on the semi-infinite slab $\left\{(x, t) \in X: t>t_{m}\right\}$ corresponding to the characteristic function of $D_{m}=\{y \in$ $\left.\mathbf{R}^{n}:\left|y-y_{m}\right|^{2}<\beta t_{m}\right\}$. Then

$$
\hat{R}_{E_{m}} 1(z, r) \geq C\left(r-t_{m}\right)^{-n / 2} \int_{D_{m}} \exp \left\{-\frac{|z-y|^{2}}{4\left(r-t_{m}\right)}\right\}\left\{1-\exp \left(-\frac{z_{n} y_{n}}{r-t_{m}}\right)\right\} d y
$$

and easy estimates give

$$
\liminf _{m \rightarrow \infty} t_{m}^{-n / 2} \hat{R}_{E_{m}} 1(z, r)>0 .
$$

Hence, $\hat{R}_{E_{m}} K_{b}(z, r) \geq C>0$, which implies that $E$ is not semithin at $b$. 
Proposition 3.2. Let $\left\{\left(y_{m}^{\prime}, y_{n, m}, t_{m}\right)\right\}$ be a sequence of points in $P(b ; \alpha: \beta)$ converging to $b=\left(b^{\prime}, 0, s\right) \in V$. For each $m$, let

$$
E_{m}=\left\{\left(x, t_{m}+y_{n, m}^{2}\right) \in X: \sigma y_{n, m}<\left|x-\left(y_{m}^{\prime}, 0\right)\right|<\nu y_{n, m},\left|x^{\prime}-y_{m}^{\prime}\right|<\delta x_{n}\right\},
$$
where $0<\sigma<\nu\left(1+\delta^{2}\right)^{-1 / 2}$ and $\alpha+\omega>0$. Then $\bigcup_{m=1}^{\infty} E_{m}$ is not semithin at $b$.

PROOF. Fix a point $(z, r) \in X_{b}^{+}$, and for each $m$ let $T_{m}=t_{m}+\omega y_{n, m}^{2}$ and $D_{m}=\left\{x:\left(x, T_{m}\right) \in E_{m}\right\}$. Then for sufficiently large $m, s<T_{m}<r$ and

$$
\hat{R}_{E_{m}} 1(z, r) \geq \int_{D_{m}} G\left(z, r ; x, T_{m}\right) d x .
$$

Now, put $\nu_{1}=\nu\left(1+\delta^{2}\right)^{-1 / 2}$. Then $\left|x^{\prime}-y_{m}^{\prime}\right|<\delta \sigma y_{n, m}, \sigma y_{n, m}<x_{n}<\nu_{1} y_{n, m}$, implies $x \in D_{m}$. Hence, $\hat{R}_{E_{m}} 1(z, r)$ dominates

$$
\frac{C}{(r-s)^{n / 2}} \exp \left\{-\frac{\left|y_{m}-z\right|^{2}+C y_{n, m}^{2}}{2(r-s)}\right\} y_{n, m}^{n-1} \int_{\sigma y_{n, m}}^{\nu_{1} y_{n, m}}\left\{1-\exp \left(-\frac{z_{n} x_{n}}{r-s}\right)\right\} d x_{n}
$$

which dominates

$$
C y_{n, m}^{n+1} y_{n, m}^{-1}\left\{1-\exp \left(-\frac{\sigma z_{n} y_{n, m}}{r-s}\right)\right\}
$$

Hence

$$
\liminf _{m \rightarrow \infty} y_{n, m}^{-(n+1)} \hat{R}_{E_{m}} 1(z, r)>0 .
$$

Now, for all $x \in D_{m}, C^{-1} y_{n, m}^{2}<T_{m}-s<C y_{n, m}^{2}, \sigma y_{n, m}<\left|x-\left(y_{m}^{\prime}, 0\right)\right|<$ $\left(1+\delta^{2}\right)^{1 / 2} x_{n}$, and $\left|x-\left(b^{\prime}, 0\right)\right|<C y_{n, m}$. Hence, $K_{b}\left(x, T_{m}\right) \geq C y_{n, m}^{-(n+1)}$, and so $\liminf _{m \rightarrow \infty} \hat{R}_{E_{m}} K_{b}(z, r)>0$.

4. Harnack inequalities. In [10], a Harnack inequality in which the limiting behavior of the Harnack constant is known was obtained for positive solutions of the heat equation on an infinite slab. Similar results are obtained in this section for solutions on the semi-infinite slab. The following technical results will be useful.

LEMMA 4.1. (i) For any $\rho>1$,

$$
|y-b|^{2}-\rho^{-1}|x-b|^{2} \geq-(\rho-1)^{-1}|x-y|^{2}
$$

for all $b, x, y \in \mathbf{R}^{n}$.

(ii) If $\rho>1$ and $\alpha<\rho \beta$, then $\rho \beta\left(1-e^{-\alpha \lambda / \rho}\right)>\alpha\left(1-e^{-\beta \lambda}\right)$ for all $\lambda \geq 0$.

ProOF. The inequality in (i) is equivalent to

$$
\rho(\rho-1)|y-b|^{2}+\rho|x-y|^{2} \geq(\rho-1)|x-b|^{2} .
$$

This follows from the triangle inequality and the fact that $\rho(\rho-1) \gamma^{2}+\rho \delta^{2} \geq$ $(\rho-1)(\gamma+\delta)^{2}$ for all $\gamma, \delta \in \mathbf{R}$.

Proposition 4.2. (i) For each $\rho>1$, there exists $\theta(\rho)>0$ such that for any nonnegative Borel measure $\mu$ on $H$ and $t>0$,

$$
\int K_{b}(x, t) d \mu(b) \geq \theta(\rho) \int K_{b}(y, t) d \mu(b)
$$

if $|x-y|^{2} \leq(\rho-1)^{2} t$ and $\rho^{-1} y_{n} \leq x_{n} \leq \rho y_{n}$. Furthermore, $\lim _{\rho \rightarrow 1} \theta(\rho)=1$. 
(ii) For each $0<\rho<1$, there exists $\varphi(\rho)>0$ such that for any nonnegative Borel measure $\mu$ on $H$ and $t>0$,

$$
\int K_{b}(x, \rho t) d \mu(b) \leq \varphi(\rho) \int K_{b}(y, t) d \mu(b) \quad \text { if }|x-y|^{2} \leq \rho^{-1}(1-\rho)^{2} t
$$

and $\rho y_{n} \leq x_{n} \leq \rho^{-1} y_{n}$. Furthermore, $\lim _{\rho \rightarrow 1} \varphi(\rho)=1$.

Proof. Let $\rho>1$ and $b \in H$. Then, by using Lemma 4.1,

$$
K_{b}(x, t) \geq \rho^{-(n+4) / 2} \exp ((1-\rho) / 4) K_{b}(y, t) .
$$

The result in (ii) follows from (i) by interchanging $x$ and $y$, replacing $t$ by $\rho t$, and setting $\varphi(\rho)=\left\{\theta\left(\rho^{-1}\right)\right\}^{-1}$.

PROPOSITION 4.3. (i) For each $0<\rho<1$ there exists $\theta_{1}(\rho)>0$ such that for any nonnegative Borel $\mu$ on $V$ and $r>0$,

$$
\int K_{b}\left(c, r+\rho(1-\rho) y_{n}^{2}\right) d \mu(b) \geq \theta_{1}(\rho) \int K_{b}(y, r) d \mu(b)
$$

if $\rho^{1 / 2} y_{n} \leq\left|x-\left(y^{\prime}, 0\right)\right| \leq y_{n},\left|x^{\prime}-y^{\prime}\right| \leq(1-\rho) x_{n}$. Furthermore, $\lim _{\rho \rightarrow 1} \theta_{1}(\rho)=1$.

(ii) For each $\rho>1$ there exists $\varphi_{1}(\rho)>0$ such that for any nonnegative Borel $\mu$ on $V$ and $r>0$,

$$
\int K_{b}\left(x, r-\rho(\rho-1) y_{n}^{2}\right) d \mu(b) \leq \varphi_{1}(\rho) \int K_{b}(y, r) d \mu(b)
$$

if $y_{n} \leq\left|x-\left(y^{\prime}, 0\right)\right| \leq \rho^{1 / 2} y_{n},\left|x^{\prime}-y^{\prime}\right| \leq(\rho-1) y_{n}$. Furthermore, $\lim _{\rho \rightarrow 1} \varphi_{1}(\rho)=1$.

Proof. Let $b=\left(b^{\prime}, 0, s\right) \in V, s<t, s<r$. Then

$$
\frac{K_{b}(x, t)}{K_{b}(y, r)}=\frac{x_{n}}{y_{n}} \tau^{(n+2) / 2} \exp \left\{\frac{1-\tau}{4(t-r)}\left(\frac{\left|y-\left(b^{\prime}, 0\right)\right|^{2}}{\tau}-\left|x-\left(b^{\prime}, 0\right)\right|^{2}\right)\right\},
$$

where $\tau=(r-s) /(t-s)$.

To prove (i), put $t=r+\rho(1-\rho) y_{n}^{2}$ and assume the conditions on $x$ and $y$ in (i) are satisfied. Then $0<\tau<1$, and so

$$
\frac{\left|y^{\prime}-b^{\prime}\right|^{2}}{\tau}-\left|x^{\prime}-b^{\prime}\right|^{2} \geq-\frac{\left|x^{\prime}-y^{\prime}\right|^{2}}{1-\tau} \geq-\frac{(1-\rho)^{2}}{1-\tau} x_{n}^{2}
$$

Hence,

$$
K_{b}(x, t) / K_{b}(y, r) \geq\left\{\rho /(1+(1-\rho))^{2}\right\}^{1 / 2} \psi(\tau)
$$

where

$$
\psi(\lambda)=\lambda^{(n+2) / 2} \exp \left\{\frac{1}{4 \rho(1-\rho)}\left[\frac{(1-\lambda)^{2}}{\lambda}-(1-\rho)^{2}\right]\right\}, \quad \text { for } \lambda>0
$$

Then

$$
\psi^{\prime}(\lambda)=\frac{\lambda^{2}+2(n+2) \rho(1-\rho) \lambda-1}{4 \lambda^{2} \rho(1-\rho)} \psi(\lambda) .
$$

Put

$$
\lambda_{1}=\left\{(n+2)^{2} \rho^{2}(1-\rho)^{2}+1\right\}^{1 / 2}-(n+2) \rho(1-\rho) .
$$

Then

$$
\psi(\lambda) \geq \psi\left(\lambda_{1}\right) \geq \lambda_{1}^{(n+2) / 2} \exp \left(-\frac{1-\rho}{4 \rho}\right), \text { for all } \lambda>0 .
$$


Put

$$
\theta_{1}(\rho)=\left\{\frac{\rho}{1+(1-\rho)^{2}}\right\}^{1 / 2} \lambda_{1}^{(n+2) / 2} \exp \left(-\frac{1-\rho}{4 \rho}\right) .
$$

To prove (ii), put $t=r-\rho(\rho-1) y_{n}^{2}$ and assume $x$ and $y$ are as in the statement of (ii). Put $\omega=1+(\rho-1)^{2}$. Then $\rho^{-1 / 2} x_{n} \leq y_{n} \leq \omega^{1 / 2} x_{n}, \tau>1$, and $K_{b}(x, t) / K_{b}(y, r) \leq \rho^{1 / 2} \chi(\tau)$, where

$$
\chi(\tau)=\tau^{(n+2) / 2} \exp \left\{\frac{1}{4 \rho(\rho-1)}\left[\rho(\rho-1)^{2}+\left(1-\frac{1}{\tau}\right)-\frac{\tau-1}{\omega}\right]\right\},
$$

for $\tau>1$. Then $\chi^{\prime}(\tau)=0$ implies $\tau^{2}-2 \rho(\rho-1)(n+2) \omega \tau-\omega=0$. Put

$$
\tau_{1}=\rho(\rho-1)(n+2) \omega+\left\{\rho^{2}(\rho-1)^{2}(n+2)^{2} \omega^{2}+\omega\right\}^{1 / 2} .
$$

Then $\chi$ attains its supremum at $\tau_{1}$. The result in (ii) follows by putting $\varphi_{1}(\rho)=$ $\rho^{1 / 2} \chi\left(\tau_{1}\right)$.

5. Semifine and parabolic limits. The precise Harnack inequalities obtained in $\S 4$ will be used here to prove the equivalence of semifine and parabolic limit at each point of $B_{0}=B \backslash\left(\mathbf{R}^{n-1} \times\{0\} \times\{0\}\right)$ for positive solutions of the heat equation.

THEOREM 5.1 (CF. [10, THEOREM 6.2]). Let $u \geq 0$ be a solution of the heat equation on $X$ having parabolic cluster value $\lambda$ at $b \in B_{0}$. Then

semifine $\lim \inf u(b) \leq \lambda \leq$ semifine $\lim \sup u(b)$.

Consequently, for any $b \in B_{0}$,

fine $\lim u(b)=\lambda \Rightarrow$ semifine $\lim u(b)=\lambda \Leftrightarrow$ parabolic $\lim u(b)=\lambda$.

ProOF. Let $b=\left(b^{\prime}, b_{n}, 0\right) \in H$. Since $\int_{V} K_{y}(x, t) d \mu(y) \rightarrow 0$ continuously on $H$ (cf. [15, p. 72]), it suffices to consider $u(x, t)=\int K_{y}(x, t) d \mu(y)$ for some Borel measure $\mu$ on $H$. Assume furthermore that $\lambda<\infty$. Then there is a sequence of points $\left\{\left(y_{m}, t_{m}\right)\right\}$ in a parabolic region $P(b ; \alpha)$ converging to $b$ such that for all $\delta>0$, there exists $M(\delta)$ such that $\lambda-\delta<u\left(y_{m}, t_{m}\right)<\lambda+\delta$ for all $m \geq M(\delta)$. For each $\rho>1$ and $m \in \mathbf{N}$, define

$$
E_{m, \rho}=\left\{\left(x, \rho t_{m}\right):\left|x-y_{m}\right|^{2} \leq(\rho-1)^{2} t_{m}\right\} .
$$

Now, choose $m_{0} \geq M(\delta)$ such that $\rho^{2} t_{m}<\left(b_{n} / 2\right)^{2}<y_{n, m}^{2}$ for all $m \geq m_{0}$. Then $\rho^{-1} y_{n, m} \leq x_{n} \leq \rho y_{n, m}$ if $\left|x-y_{m}\right|^{2} \leq(\rho-1)^{2} t_{m}$. Hence by Proposition 4.2, $u(x, t) \geq \theta(\rho) u\left(y_{m}, t_{m}\right)>\lambda-2 \delta$ for all $m \geq m_{0}$ and $(x, t) \in E_{m, \rho}$, for some $\rho$, since $\theta(\rho) \rightarrow 1$. Therefore $u>\lambda-2 \delta$ on the set $\bigcup_{m \geq m_{0}} E_{m, \rho}$, which is not semithin at $b$, by Proposition 3.1. Hence $\lambda \leq$ semifine lim sup $u(b)$. The other inequality is proved in a similar manner.

To complete the proof in case $b \in H$, consider $\lambda=\infty$. Fix $\rho>1$ and let $\left\{\left(y_{m}, t_{m}\right)\right\}$ converge to $b$ in $P(b ; \alpha)$ and $u\left(y_{m}, t_{m}\right) \rightarrow \infty$. Then for any $\delta>0$, choose $M(\delta)$ such that $u\left(y_{m}, t_{m}\right)>\delta[\theta(\rho)]^{-1}$ for all $m \geq M(\delta)$. Now, choose $m_{0} \geq M(\delta)$ as above. Then $u>\delta$ on a set which is not semithin at $b$.

Now assume $b \in V \cap B_{0}$. It is easy to show that $\int_{H} K_{y}(x, t) d \mu(y) \rightarrow 0$ continuously on $V \cap B_{0}$. Hence it suffices to consider $u(x, t)=\int K_{y}(x, t) d \mu(y)$ for 
some Borel measure $\mu$ on $V$. Let $\left\{\left(y_{m}, t_{m}\right)\right\}$ converge to $b$ in $P(b ; \alpha: \beta)$ such that $u\left(y_{m}, t_{m}\right) \rightarrow \lambda$.

$$
\begin{aligned}
E_{m, \rho}=\left\{\left(x, t_{m}+\rho(1-\rho) y_{n, m}^{2}\right): \rho^{1 / 2} y_{n, m} \leq\right. & \left|x-\left(y_{m}^{\prime}, 0\right)\right| \leq y_{n, m}, \\
& \left.\left|x^{\prime}-y_{m}^{\prime}\right| \leq(1-\rho) x_{n}\right\} .
\end{aligned}
$$

Then Proposition 4.3 implies that $u(x, t) \geq \theta_{1}(\rho) u\left(y_{m}, t_{m}\right)$ for all $m \in \mathbf{N}$ and $(x, t) \in E_{m}$. Now, $\rho\left\{1+(1-\rho)^{2}\right\}<1$ and $\alpha+\rho(1-\rho)>0$. Hence Proposition 3.2 implies that $\bigcup_{m \geq m_{0}} E_{m, \rho}$ is not semithin at $b$.

For each $\rho>1, m \in \mathbf{N}$ define

$$
\begin{array}{r}
F_{m, \rho}=\left\{\left(x, t_{m}-\rho(\rho-1) y_{n, m}^{2}\right): y_{n, m} \leq\left|x-\left(y_{m}^{\prime}, 0\right)\right| \leq \rho^{1 / 2} y_{n, m},\right. \\
\left.\left|x^{\prime}-y_{m}^{\prime}\right| \leq(\rho-1) x_{n}\right\} .
\end{array}
$$

Then $u(x, t) \leq \varphi_{1}(\rho) u\left(y_{m}, t_{m}\right)$ for all $(x, t) \in F_{m, \rho}$. Now, there exists $\rho_{0}, 1<\rho_{0}<$ 2 , such that $1<\rho<\rho_{0}$ implies $1+(\rho-1)^{2}<\rho$ and $\alpha-\rho(\rho-1)>0$. Hence, Proposition 3.2 implies that $\bigcup_{m \geq m_{0}} F_{m, \rho}$ is not semithin at $b$ if $1<\rho<\rho_{0}$. The proof is completed as above.

Next, an example of a positive solution is given which has neither fine, semifine, nor parabolic limits at any point of $B \backslash B_{0}$. Hence there is no advantage in trying to extend the above result to points on $B \backslash B_{0}$.

EXAMPLE 5.2. Let $u$ be the solution represented by Lebesgue measure restricted to $H$. Then

$$
u(x, t)=\frac{2}{\sqrt{\pi}} \int_{0}^{x_{n} / 2 \sqrt{t}} e^{-r^{2}} d r .
$$

For each $0<\tau<1$, let $\alpha_{\tau}$ be such that $(2 / \sqrt{\pi}) \int_{0}^{\alpha_{\tau}} e^{-r^{2}} d r=\tau$. For each $\varepsilon>0$ and $\lambda \geq 0,|u(x, t)-\lambda|>\varepsilon$ iff $\tau<u(x, t)<\omega$, where $\tau=\max (\lambda-\varepsilon, 0)$ and $\omega=$ $\min (\lambda+\varepsilon, 1)$ iff $2 \tau \sqrt{t}<x_{n}<2 \omega \sqrt{t}$. Hence, by Proposition 3.2, $\{(x, t): \mid u(x, t)-$ $\lambda \mid>\varepsilon\}$ is not semithin (hence not thin) at each $b \in B \backslash B_{0}$. Also, it is easy to see that $u$ does not have a parabolic limit at points on $B \backslash B_{0}$.

6. Parabolic and fine limits almost everywhere. In $\S 5$ it was proved that for positive solutions semifine and parabolic limits are equivalent at each point of $B_{0}$. This section shows that fine and parabolic limits are equivalent except on a set of measure zero for positive solutions.

LEMMA 6.1. Let $E \subset B$ and $W \subset X$ be such that for each $b \in E, W$ contains $a$ truncated parabolic region with vertex $b$. Then $X \backslash W$ is thin at almost every $b \in E$.

Proof. Let $E_{1}=E \cap H$ and $E_{2}=E \cap V$. As in the proof of [10, Lemma 5.1], it suffices to assume that $E_{1}$ and $E_{2}$ are compact, $\operatorname{dist}\left(E_{1}, V\right)>0, \operatorname{dist}\left(E_{2}, H\right)>0$, $W_{1} \supset \bigcup_{b \in E_{1}} P(b ; \alpha: \delta)$, and $W_{2} \supset \bigcup_{b \in E_{2}} P(b ; \tau: \nu) \cap\left\{(x, t): x_{n}<\delta\right\}$.

Now, define $G_{1}=\left(X \backslash W_{1}\right) \cap\{(x, t): t<\delta\}$ and $G_{2}=\left(X \backslash W_{2}\right) \cap\left\{(x, t): x_{n}<\delta\right\}$. Then, it suffices to prove that $G_{i}$ is thin at a.e. $b \in E_{i}$. To do this, let $u_{i}$ be the solution of the Dirichlet problem on $X$ corresponding to the characteristic function of $E_{i}$. Then for any $0<\lambda<1$ and $i=1,2$, the set $F_{i}(\lambda)=\left\{(x, t) \in X: u_{i}(x, t) \leq\right.$ $\lambda\}$ is thin at a.e. $b \in E_{i}$. Let $D_{i}=\left\{b \in E_{i}:(x, t) \in P(b ; \alpha)\right\}, i=1,2$. Then $(x, t) \in G_{i} \Rightarrow E_{i} \subset B \backslash D_{i}, K_{b}(x, t) \geq C t^{-n / 2}$ if $b \in D_{1}$, and $K_{b}(x, t) \geq C x_{n}^{-(n+1)}$ if $b \in D_{2}$, where $C$ is independent of $(x, t)$ and $b$. By assuming that $\operatorname{dist}\left(W_{1}, V\right)>0$, 
it follows that the volume of $D_{1} \geq C t^{n / 2}$. Also, in case of $D_{2}$, choose $\alpha=\tau: \nu$ such that $\tau^{2}\left(1+\nu^{-2}\right)<1$; then

$$
\left\{\left(b^{\prime}, 0, s\right): \tau\left(1+\nu^{-2}\right) x_{n}^{2} \leq t-s \leq \tau^{-1} x_{n}^{2}, x_{n} \geq \nu\left|x^{\prime}-b^{\prime}\right|\right\} \subset D_{2} .
$$

Hence the volume of $D_{2} \geq C x_{n}^{n+1}$. Consequently, $u_{i}(x, t) \leq 1-C<1$.

The main result of this section can now be obtained by suitably modifying the proof of Theorem 5.2 in [10].

THEOREM 6.2. If $f$ has parabolic limit $\psi(b)$ at each $b \in E$, then fine $\lim f(b)=$ $\psi(b)$ for a.e. $b \in E$.

7. A local Fatou theorem. Theorem 9.2 in [10] already gives a Carleson-type local Fatou theorem for sets which touch the horizontal part of the boundary $B$. So to establish the analogue of Theorem 9.2 of [10] here, one only needs to consider the vertical boundary $V$.

By using Theorem 1.1, Moser's Harnack inequality (cf. [12]), and the nonthin set constructed in [9], the next result can be proved by an obvious modification of Theorem 4.2 in [9].

THEOREM 7.1. Let $b \in V$ and $u \geq 0$ be a solution of the heat equation on $T P(b ; \tau: \delta, \rho)$. Let $\alpha>\tau$ and $\beta>\delta$. If $u$ has limit 0 along the fine filter $\mathcal{F}(b)$ restricted to $T P(b ; \alpha: \beta)$, then $u(x, t) \rightarrow 0$ as $(x, t) \rightarrow b$ within $T P(b ; \alpha: \beta)$.

LEMMA 7.2. Let $E \subset V$ and $W \subset X$ be such that for each $b \in E, W$ contains a two-sided parabolic region with vertex $b$. Then for a.e. $b \in E, W$ contains two-sided parabolic regions of arbitrary aperture with vertex $b$.

Proof. It suffices to assume that $\operatorname{dist}(E, H)>0$ and $W=\bigcup_{b \in E} T P(b ; \alpha: \beta, \delta)$ for fixed $\alpha, \beta, \delta>0$. Choose $m_{0}$ such that $1 / m_{0}<\delta$. Fix $\nu, \rho>0$. For each $m \geq m_{0}$, define $E_{m}=\{b \in E: T P(b ; \nu: \rho, 1 / m) \subset W\}$. Let $D$ be the set of points of strong density of $E$. As in [10, Lemma 9.1], it suffices to prove that $D \subset \bigcup_{m \geq m_{0}} E_{m}$. If $b=\left(b^{\prime}, 0, s\right) \notin E_{m}$ for $m \geq m_{0}$, there exists a sequence $\left\{\left(x_{m}, t_{m}\right)\right\}$ contained in $T P(b ; \nu: \rho) \backslash W$ such that $\left|x_{m}-\left(b^{\prime}, 0\right)\right|<1 / m$.

Define

$$
F_{m}=\left\{\left(y^{\prime}, 0, p\right):\left|p-t_{m}\right| \leq \alpha x_{n, m}^{2},\left|y^{\prime}-x_{m}^{\prime}\right| \leq \beta x_{n, m}\right\} .
$$

Then $F_{m} \cap E=\varnothing$ for all $m \geq m_{0}$. Now, $\left(x_{m}, t_{m}\right) \in T P(b ; \nu: \rho)$. Hence $\left|t_{m}-s\right| \leq$ $\nu\left(1+\rho^{2}\right) x_{n, m}^{2}$ and $\left|x_{m}^{\prime}-b^{\prime}\right| \leq \rho x_{n, m}$. Therefore, $y=\left(y^{\prime}, 0, p\right) \in F_{m}$ implies that $|p-s| \leq\left[\alpha+\nu\left(1+\rho^{2}\right)\right] x_{n, m}^{2}$ and $\left|y^{\prime}-b^{\prime}\right| \leq(\beta+\rho) x_{n, m}$. Hence the rectangle $F_{m}$ is contained within another rectangle of comparable volume having center $b$.

THEOREM 7.3 (CF. [10, THEOREM 9.2]). Let $E \subset B$ and $W$ be an open subset of $X$ which contains a region $W_{b}$ for each $b \in E$ where $W_{b}$ is a parabolic region with vertex $b$ for each $b \in E \cap H$ and $W_{b}$ is a two-sided parabolic region with vertex $b$ for each $b \in E \cap V$. Let $u$ be a solution of the heat equation on $W$ which is either upper or lower bounded on $W_{b}$ for each $b \in E$. Then $u$ has finite two-sided parabolic limits a.e. on $E$.

PROOF. It suffices to assume $E$ compact $\subset V \cap B_{0}, \alpha, \beta, \delta$ fixed and $W_{b}=$ $T P(b ; \alpha: \beta, \delta)$ for all $b \in E, W$ connected and $u>1$ on $W$. By suitable truncations of $W$ by horizontal planes, it is seen that condition $(* *)$ in $[\mathbf{1 0}$, p. 592] is satisfied 
by $W$. It is also clear that there is a constant $\tau>0$ such that the plane $x_{n}=\tau$ intersects $W_{b}$ for all $b \in E$. Let $A=W \cap\left\{(x, t): x_{n}=\tau\right\}$, and let $l_{n}$ denote Lebesgue $n$-dimensional measure on $A$.

Define the measure $r$ on $X$ by $r=u^{-1} l_{n}$. Then $r$ is supported on $W$ and $s=\left.r\right|_{W}=r$. Clearly, $r(X)<\infty$, and the functions 1 and $u$ are $s$-integrable. Also, $r$ is a reference measure on $W$ and on $X$ (cf. [10, Theorem 9.2]). For each $b \in B_{0}$, define $Q(b)=\int K_{b} d r$ as in [10]. Then $0<Q(b)<\infty$, and $Q$ is continuous.

Define $\Omega: B_{0} \rightarrow B_{r}(X)=\left\{u \geq 0: u\right.$ is minimal solution, $\left.\int u d r=1\right\}$, by $\Omega(b)=Q(b)^{-1} K_{b}$. Then $\Omega$ is continuous and injective. It does not seem that $\Omega$ is a homeomorphism (cf. [10, Theorem 9.2]); however, the representing measure for the constant function 1 on $X$ can still be related to Lebesgue measure on $B_{0}$ as follows.

Let $\nu$ be the measure on $B_{0}$ defined by $d \nu(b)=Q(b) d b$. Then $\nu\left(B_{0}\right)=$ $\int_{B_{0}} Q(b) d b=r(X)$ by Fubini. Let $\nu_{1}=\nu \circ \Omega^{-1}$. Then

$$
\int_{B_{r}(x)} k(x, t) d \nu_{1}(k)=\int_{B_{0}} K_{b}(x, t) d b=1 .
$$

Hence $\nu_{1}$ is the representing measure for 1 on $B_{r}(x)$. Now, from Lemmas 6.1 and 7.2 there is a set $E_{1} \subset E$ such that $E \backslash E_{1}$ is of Lebesgue measure zero, $X \backslash W$ is thin at every $b \in E_{1}$, and for each $b \in E_{1}, W$ contains two-sided parabolic regions of arbitrary aperture with vertex $b$. The proof is completed as in [10, Theorem 9.2] by using Theorem 7.1; and the reduction theorem and the local fine limit theorem in $[\mathbf{1 0}]$.

\section{REFERENCES}

1. H. Bauer, Harmonische Raume und ihre Potentialtheorie, Lecture Notes in Math., vol. 22, Springer-Verlag, New York and Berlin, 1966.

2. M. Brelot and J. L. Doob, Limites angulaires et limites fines, Ann. Inst. Fourier (Grenoble) 13 (1963), 395-415.

3. A. P. Calderon, On the behaviour of harmonic functions at the boundary, Trans. Amer. Math. Soc. 68 (1950), 47-54.

4. L. Carleson, On the existence of boundary values for harmonic functions in several variables, Ark. Mat. 4 (1961), 393-399.

5. E. B. Fabes, N. Garaofalo and S. Salsa, A backward Harnack inequality and Fatou theorem for non-negative solutions of parabolic equations, Univ. of Minnesota Math. Rep. 83-117.

6. B. F. Jones, Jr. and C. C. Tu, Non-tangential limits for a solution of the heat equation in a two-dimensional Lip $\alpha$ region, Duke Math. J. 37 (1970), 243-254.

7. R. Kaufman and J.-M. Wu, Parabolic potential theory, J. Differential Equations 43 (1982), 204-234.

8. J. T. Kemper, Temperatures in several variables: kernel functions, representations, and parabolic boundary values, Trans. Amer. Math. Soc. 167 (1972), 243-262.

9. A. Koranyi and J. C. Taylor, Fine convergence and parabolic convergence for the Helmholtz equation and the heat equation, Illinois J. Math. 27 (1983), 77-93.

10. B. A. Mair, Fine and parabolic limits for solutions of second-order linear parabolic equations on an infinite slab, Trans. Amer. Math. Soc. 284 (1984), 583-599.

11. B. A. Mair and J. C. Taylor, Integral representation of positive solutions of the heat equation, Lecture Notes in Math., Springer-Verlag, vol. 1096, pp. 419-433.

12. J. Moser, A Harnack inequality for parabolic differential equations, Comm. Pure Appl. Math. 17 (1964), 101-134. 
13. J. C. Taylor, An elementary proof of the theorem of Fatou-Naim-Doob, 1980 Seminar on Harmonic Analysis (Montreal, Que., 1980), Canad. Math. Soc. Conf. Proc., vol. 1, Amer. Math. Soc., Providence, R. I., 1981, pp. 153-163.

14. C. C. Tu, Non-tangential limits of a solution of a boundary-value problem for the heat equation, Math. Systems Theory 3 (1969), 130-138.

15. D. V. Widder, The heat equation, Academic Press, New York, 1975.

Department of Mathematics, The Pennsylvania State University, Mont alto Campus, Mont alto, Pennsylvania 17237 